\title{
EFFECT OF RESIN INFILTRANT AND FLUORIDE VARNISH ON MICRO-HARDNESS OF DE-MINERALIZED ENAMEL SUBMITTED TO pH CHALLENGE
}

\author{
Reham M. Attia*
}

\begin{abstract}
The purpose of this study was to evaluate the micro-hardness of artificially induced demineralized enamel after application of resin infiltrant and fluoride varnish and after $\mathrm{pH}$ challenge.

Material and methods: In the current study; sound bovine incisors were used. A total of 40 sound enamel were then embedded in pre-cut metal cylinders. Two layers of acid-resistant nail varnish were applied to cover most of the enamel surfaces, leaving a window of $4 \times 4 \mathrm{~mm}$ for demineralization. Each specimen was immersed in $32 \mathrm{ml}$ of a de-mineralizing solution containing $50 \mathrm{mM}$ acetate buffer solution and $1.28 \mathrm{mM} \mathrm{Ca}(\mathrm{NO} 3) 2 \_4 \mathrm{H} 2 \mathrm{O}, 0.74 \mathrm{mM}$ (NaH2PO4)_2H2O, and $0.03 \mathrm{ppm} \mathrm{F}$ at pH 5.0,10 for 24 hours at $37^{\circ} \mathrm{C}$, then microhardness was recorded. The samples then were divided into two groups according to the material used to treat the de-mineralized enamel, each group consisted of 20 samples. Group 1: The samples of de-mineralized enamel were infiltrated with resin infiltration. Then the micro-hardness was recorded for all resin infiltrated samples before $\mathrm{pH}$ cycling challenge. The samples were then submitted to a $\mathrm{pH}$ cycling model at $37 \mathrm{C}$ over 7 days . The $\mathrm{pH}$ cycling consisted of immersing the samples in $35.5 \mathrm{ml}$ of de-mineralizing solution: (2.0 $\mathrm{mmol} / \mathrm{L} \mathrm{Ca}, 2.0 \mathrm{mmol} / \mathrm{L} \mathrm{P}, 0.075 \mathrm{~mol} / \mathrm{L}$ acetate buffer, $2.22 \mathrm{~mL} / \mathrm{mm}^{2}$ of enamel surface) for 6 hours, alternated with immersion in $17.75 \mathrm{~mL}$ of re-mineralizing solution: $(1.5 \mathrm{mmol} / \mathrm{L} \mathrm{Ca}, 0.9$ $\mathrm{mmol} / \mathrm{L} \mathrm{P}, 0.15 \mathrm{~mol} / \mathrm{L} \mathrm{KCl}, 0.02 \mathrm{~mol} / \mathrm{L}$ cacodylate buffer, $\mathrm{pH} 7.0,0.25 \mathrm{~mL} / \mathrm{mm}^{2}$ ) for 18 hours for 5 days. Then, specimens were kept for 2 more days in a fresh re-mineralizing solution, completing 7 days of treatment. The samples were washed in de-ionized water for 30 seconds among demineralizing and re-mineralizing cycles. Group 2: Fluoride varnish (Duraphat, Colgate- Palmolive ,NSW, Australia) was applied as a thin layer by a brush and totally dried, then the micro-hardness was recorded. The samples were then subjected to a $\mathrm{pH}$ cycling as in group 1 . Then after challenge the micro-hardness measurements were performed as formerly described.
\end{abstract}

Results: The difference was highly significant between resin infiltrant and fluoride varnish treated enamel. The comparison among micro-hardness values of initial, de-mineralized enamel, resin-infiltrated enamel and resin infiltrated after $\mathrm{pH}$ cycling showed that there was a significant difference. The difference between micro-hardness values was highly significant when comparison was accomplished between initial and de-mineralized enamel, initial and after $\mathrm{pH}$ cycling, demineralized enamel and resin infiltrated enamel, de-mineralized enamel and after $\mathrm{pH}$ cycling and finally between resin infiltrated enamel and after $\mathrm{pH}$ cycling. The difference between microhardness values was not significant regarding initial and resin infiltrated enamel.

Conclusion: Within the limitations of this study, it was concluded that the micro-hardness of resin infiltrated enamel was higher than that of de-mineralized enamel treated with fluoride varnish before and after $\mathrm{pH}$ cycling.

* Lecturer of Operative Dentistry,Department of Conservative Dentistry, Faculty of Dentistry, Zagazig University, Egypt 


\section{INTRODUCTION}

The main goal in modern dentistry is the early detection, protection and re-mineralization of incipient lesions ${ }^{[1,2]}$. Traditional approaches with extensive restorations have changed to prevention, noninvasive, and minimal invasive methods. Recent procedures of controlling caries, including preventive methods, risk control, and early diagnosis of caries, can help to stop these early enamel lesions at the early stages. Recently, tooth decay control methods have changed significantly ${ }^{[2]}$.

One of the noninvasive treatments is the remineralization with fluoride varnish. It is well confirmed that fluoride varnish is effective in preventing tooth decay ${ }^{[3]}$. Fluoride varnish can improve and enhance the results of topical fluoride therapies by increasing the duration of exposure to the enamel ${ }^{[4]}$. Anti-decay activity of fluoride varnish is related to its fluoride release, promoting the remineralization of enamel and preventing the enamel demineralization process ${ }^{[5]}$. Due to safety and effectiveness of fluoride, its use has been increasing in recent years ${ }^{[6]}$.

Although frequent application of fluoride is often recommended as the treatment of choice for initial enamel caries on smooth or proximal surfaces, the effectiveness of this approach depends strongly on the patient's oral hygiene practice. Therefore, it is not suitable for noncompliant patients ${ }^{[7,8]}$.

More recently, a noninvasive alternative treatment was proposed, based on caries infiltration with a hydrophobic resin. These "infiltrant" materials occlude the micro-pore structures found in de-mineralized enamel and block the passages that bacteria and acid require to cause further dissolution of the enamel structure, thus inhibiting lesion progression ${ }^{[9,}{ }^{10]}$. Caries infiltration is considered a noninvasive treatment option for noncavitated enamel lesions extending to the outer third of dentin. These lesions are not expected to show re-mineralization or arrest by other ways of noninvasive treatments ${ }^{[2]}$.
In contrast to conventional resin-based restorative materials, currently available infiltration resin is composed mainly of hydrophilic triethyleneglycoldimethacrylate (TEGDMA) ${ }^{[11,12]}$. Infiltration resin has two major differences from the other resin-based materials: it is an unfilled liquid resin composed of mostly TEGDMA and it does not have a polishing step after its application per the manufacturer's instructions. TEGDMA is important for maintaining the extremely low viscosity that allows penetration of the resin to the de-mineralized lesion ${ }^{[13,}{ }^{14]}$. However, it is well known that TEGDMA has a high water sorption rate ${ }^{[15]}$.

The infiltrant resin has a refractive index close to that of sound enamel, therefore masking the white spot by infiltrating the porous enamel ${ }^{[16]}$. This treatment has also been proposed to inhibit demineralization because the diffusion pathways for cariogenic acids are blocked, therefore sealing the white spot lesions ${ }^{[17]}$. The infiltrant resin is a product that allows for the treatment of carious lesions in early stages without invasive measures ${ }^{[18]}$.

The resin infiltration technique has many advantages such as providing mechanical stabilization for the de-mineralized enamel structure, no structure loss of the affected or neighboring teeth, occlusion of the micro pore structures in the body of the lesion, arresting or decreasing lesion progression, reducing secondary caries, delaying the need for a restoration, no postoperative sensitivity or pulp inflammation, reduction of gingivitis and periodontitis risk and good esthetic results in masking white spot ${ }^{[19,20]}$.

Bovine teeth widely used in experimental study as an substitute for human teeth due to the likeness between the two types of teeth in terms of chemical and physical properties, as its composition and hardness ${ }^{[21]}$. In addition, the composition of bovine teeth show less variations than human one, so the use of bovine teeth results in more standardized test conditions [22]. The chemical structure of bovine enamel analogous to human enamel, and 
the relatively large size of bovine teeth helps in providing sufficient enamel surfaces ${ }^{[3]}$.

Hardness means the resistance of a material or a surface against indentation or penetration. It is considered an important mechanical characteristic of the material because the resistance to abrasion, friction, erosion, water or any other substance, in general, increases with increasing hardness. This means that high levels of mineral content in enamel are associated with low levels of abrasion compared with dentin ${ }^{[23]}$.

The oral environment is constantly under mechanical and chemical challenges that may affect the tooth structure or restorative materials. $\mathrm{pH}$ cycling models are used to evaluate treatment options for white spot lesions and early carious lesion, since these models mimic $\mathrm{pH}$ alterations of the oral environment ${ }^{[24,25]}$.

It is generally accepted that all resin-based dental materials degrade to some extent in the oral environment. Surface degradation affects the mechanical properties of resin-based dental materials ${ }^{[2,26]}$. Surface micro hardness of many resin composites decrease following different challenges as $\mathrm{pH}$ changes ${ }^{[2]}$. Plasticization of the resin matrix by water sorption and hydrolytic breakdown of the resin-filler interface were considered to be the causes of the reduced surface hardness of resin composite materials ${ }^{[9]}$.

Micro hardness of enamel is decreased markedly after demineralization. One of the primary functions of any material used in management of early carious lesion is attained physical properties of sound enamel.

\section{MATERIAL AND METHODS:}

In the current study; sound permanent bovine incisors were used. They were collected from steers aged 24 to 30 months old. They were stored in $0.1 \%$ thymol solution then cleaned using slurry pumice and brush. All surfaces of the samples were examined using a stereomicroscope (SZ-CTY Olympus, Japan) to ensure that there were no cracks or other surface defects ${ }^{[2]}$.

The tooth roots were removed under water irrigation. They were first sectioned to remove their buccal surfaces using a water cooled diamond saw (Imicryl, Konya, Turkey), giving one enamel specimen per tooth. A total of 40 sound enamel were then embedded in pre-cut metal cylinders using cold acrylic resin (Imicryl, Konya, Turkey) [27]. The enamel surfaces were flattened and polished using 400-, 600-, 800-, and 1200-grit sandpaper [28]. The initial Vicker's hardness number (VHN) of each enamel surface in a sample was measured on four different points with a micro-indentation hardness tester (Zwick.roell, system 5153. UK) that was fitted with a 50-g load for 15 seconds [10].

Two layers of acid-resistant nail varnish (nail polish, Amanda, Egypt) were applied to cover most of the enamel surfaces, leaving a window of $4 \times 4 \mathrm{~mm}$ for demineralization. Each specimen was immersed in $32 \mathrm{ml}$ of a de-mineralizing solution containing $50 \mathrm{mM}$ acetate buffer solution and $1.28 \mathrm{mM}$ $\mathrm{Ca}(\mathrm{NO} 3)^{2} \_4 \mathrm{H} 2 \mathrm{O}, 0.74 \mathrm{mM}$ (NaH2PO4)_2H2O, and $0.03 \mathrm{ppm} \mathrm{F}$ at $\mathrm{pH} 5.0,10$ for 24 hours at $37 \mathrm{C}$. Then, specimens were removed from the solution and carefully washed with deionized water [2]. This treatment leads to demineralization of the enamel surface. Micro-hardness measurements of demineralized enamel surface were recorded for each sample.

The samples then were divided into two groups according to the material used to treat the demineralized enamel, each group consisted of 20 samples.

\section{Group 1:}

The samples of de-mineralized enamel were infiltrated with resin infiltration according to the manufacturer's instructions. Hydrochloric acid gel (15\%) (Icon-Etch, DMG, Hamburg, Germany) was 
applied to the surface of de-mineralized enamel for 2 minutes, and afterwards it was rinsed with water and dried in air for 30 seconds previous to ethanol (Icon-Dry, DMG, Hamburg, Germany) was applied for. The sample was then subsequently air dried .A resin infiltrant (Icon-Infiltrant, DMG, Hamburg, Germany) was then applied to the surface for 3 minutes and the sample was light-cured for 40 seconds (Cromalux curing unit. MEGA-PHYSIK DENTAL.D-76437 Rastatt. No.7050. Germany).

The resin infiltrant was reapplied for 1 minute, then the sample light-cured ${ }^{[29]}$.

The samples were polished by medium, fine, and superfine aluminum oxide Sof-Lex discs (3M-ESPE Dental Products, USA) in a low-speed hand piece under air cooling for 20 seconds. The samples were stored for 7 days at $37^{\circ} \mathrm{C}$.Then the micro-hardness were recorded for all resin infiltrated samples before $\mathrm{pH}$ cycling challenge .

The samples were then submitted to a $\mathrm{pH}$ cycling model at $37 \mathrm{C}$ over 7 days. The $\mathrm{pH}$ cycling consisted of immersing the samples in $35.5 \mathrm{ml}$ of de-mineralizing solution: $(2.0 \mathrm{mmol} / \mathrm{L} \mathrm{Ca}, 2.0$ $\mathrm{mmol} / \mathrm{L} \mathrm{P}, 0.075 \mathrm{~mol} / \mathrm{L}$ acetate buffer, $2.22 \mathrm{~mL} /$ $\mathrm{mm} 2$ of enamel surface) for 6 hours, alternated with immersion in $17.75 \mathrm{~mL}$ of re-mineralizing solution: $(1.5 \mathrm{mmol} / \mathrm{L} \mathrm{Ca}, 0.9 \mathrm{mmol} / \mathrm{L} \mathrm{P}, 0.15 \mathrm{~mol} / \mathrm{L} \mathrm{KCl}$, $0.02 \mathrm{~mol} / \mathrm{L}$ cacodylate buffer, $\mathrm{pH} 7.0,0.25 \mathrm{~mL} / \mathrm{mm}^{2}$ ) for 18 hours for 5 days. Then, specimens were kept for 2 more days in a fresh re-mineralizing solution, completing 7 days of treatment. The samples were washed in de-ionized water for 30 seconds among de-mineralizing and re-mineralizing cycles ${ }^{[30,31]}$.

\section{Group 2:}

Fluoride varnish ( Duraphat, Colgate- Palmolive, NSW, Australia) was applied as a thin layer by a brush and totally dried, then the micro-hardness was recorded ${ }^{[32]}$. The samples were then subjected to a $\mathrm{pH}$ cycling as in group 1 . Then after challenge the micro-hardness measurements were performed as formerly described. The data were collected and statistically analyzed using Statistical Package for the Social Sciences (SPSS Inc, Chicago, IL, USA) version 19.

\section{RESULTS:}

The measured micro-hardness values were recorded as baseline (sound enamel), after demineralization, after treatment of de-mineralized enamel (infiltrated or fluoride varnish treated) and after $\mathrm{pH}$ cycling challenge for both groups. Comparison between group 1 and group 2 was carried out using $\mathrm{t}$ test.

Statistical analysis of mean micro-hardness values of sound (baseline) and demineralised enamel revealed that there was no significant difference between group 1 and group 2, whereas the difference was highly significant between resin infiltrant and fluoride varnish treated enamel (table 1).

TABLE (1) Comparison of micro-hardness between group 1 and group 2 at different experimental steps.

\begin{tabular}{|c|c|c|c|c|c|c|c|c|c|}
\hline \multirow{2}{*}{\multicolumn{2}{|c|}{ Experimental steps }} & \multicolumn{6}{|c|}{ Groups } & \multicolumn{2}{|c|}{ T-Test } \\
\hline & & \multicolumn{3}{|c|}{ Group I } & \multicolumn{3}{|c|}{ Group II } & $\mathbf{t}$ & P-value \\
\hline \multirow{2}{*}{ Initial } & Range & 312.7 & - & 358 & 309 & - & 353 & \multirow{2}{*}{-0.465} & \multirow{2}{*}{0.645} \\
\hline & Mean \pm SD & 332.81 & \pm & 12.02 & 334.78 & \pm & 13.99 & & \\
\hline \multirow{2}{*}{ De-mineralized enamel } & Range & 200 & - & 255 & 200 & - & 253 & \multirow{2}{*}{0.223} & \multirow{2}{*}{0.825} \\
\hline & Mean \pm SD & 226.21 & \pm & 15.86 & 224.97 & \pm & 18.48 & & \\
\hline \multirow{2}{*}{ Fluoride varnish } & Range & 300 & - & 350.5 & 209 & - & 300.5 & \multirow{2}{*}{14.952} & \multirow{2}{*}{$<0.001^{*}$} \\
\hline & Mean \pm SD & 324.87 & \pm & 13.93 & 239.16 & \pm & 20.75 & & \\
\hline \multirow{2}{*}{$\begin{array}{c}\text { Fluoride varnish and PH } \\
\text { cycle }\end{array}$} & Range & 101 & - & 181 & 89 & - & 100.3 & \multirow{2}{*}{6.611} & \multirow{2}{*}{$<0.001^{*}$} \\
\hline & Mean \pm SD & 124.14 & \pm & 18.65 & 95.24 & \pm & 3.93 & & \\
\hline
\end{tabular}


Table 2 shows the changes in micro-hardness values in the group 1 where the resin infiltration was used at different times of micro-hardness measurements.

The comparison among micro-hardness values of initial, de-mineralized enamel, resin infiltrated enamel and resin infiltrated after $\mathrm{pH}$ cycling using Anova test revealed that there was a significant difference $(\mathrm{p}<0.001)$.

TUKEY'S Test revealed that the difference between micro-hardness values was highly significant when comparison was accomplished between initial and de-mineralized enamel, initial and after $\mathrm{pH}$ cycling, de-mineralized enamel and resin infiltrated enamel, de-mineralized enamel and after $\mathrm{pH}$ cycling and finally between resin infiltrated enamel and after $\mathrm{pH}$ cycling where $\mathrm{p}<0.001$. The difference between micro-hardness values was not significant regarding initial and resin infiltrated enamel using TUKEY'S Test where $\mathrm{p}=0.387$.

Mean and standard deviation of micro-hardness values in group 2 where the fluoride varnish was used to treat the demineralised enamel at different times of micro-hardness measurements is shown in Table 3.
Anova test revealed that there was a significant difference among micro-hardness values of initial, de-mineralized enamel, resin infiltrated enamel and resin infiltrated after $\mathrm{pH}$ cycling where $(\mathrm{p}<0.001)$.

Despite the values of micro-hardness of enamel after fluoride varnish application were higher than that of de-mineralized enamel, TUKEY'S Test revealed that the difference was not significant $(\mathrm{p}=0.033)$. The same test revealed that the difference between micro-hardness values was highly significant when comparison was accomplished between initial and de-mineralized enamel ,initial and after $\mathrm{pH}$ cycling, and de-mineralized enamel and after $\mathrm{pH}$ cycling and finally between fluoride varnish treated enamel and after $\mathrm{pH}$ cycling where $\mathrm{p}<0.001$.

TUKEY'S Test showed that the difference between micro-hardness values was highly significant. Comparison was accomplished between initial and de-mineralized enamel, initial and after $\mathrm{pH}$ cycling , de-mineralized enamel and resin infiltrated enamel, de-mineralized enamel and after $\mathrm{pH}$ cycling and finally between resin infiltrated enamel and after $\mathrm{pH}$ cycling where $\mathrm{p}<0.001$. The difference between micro-hardness values was not significant regarding initial and resin infiltrated enamel using TUKEY'S Test where $\mathrm{p}=0.387$.

TABLE (2) Comparison of micro-hardness among different experimental steps of group 1.

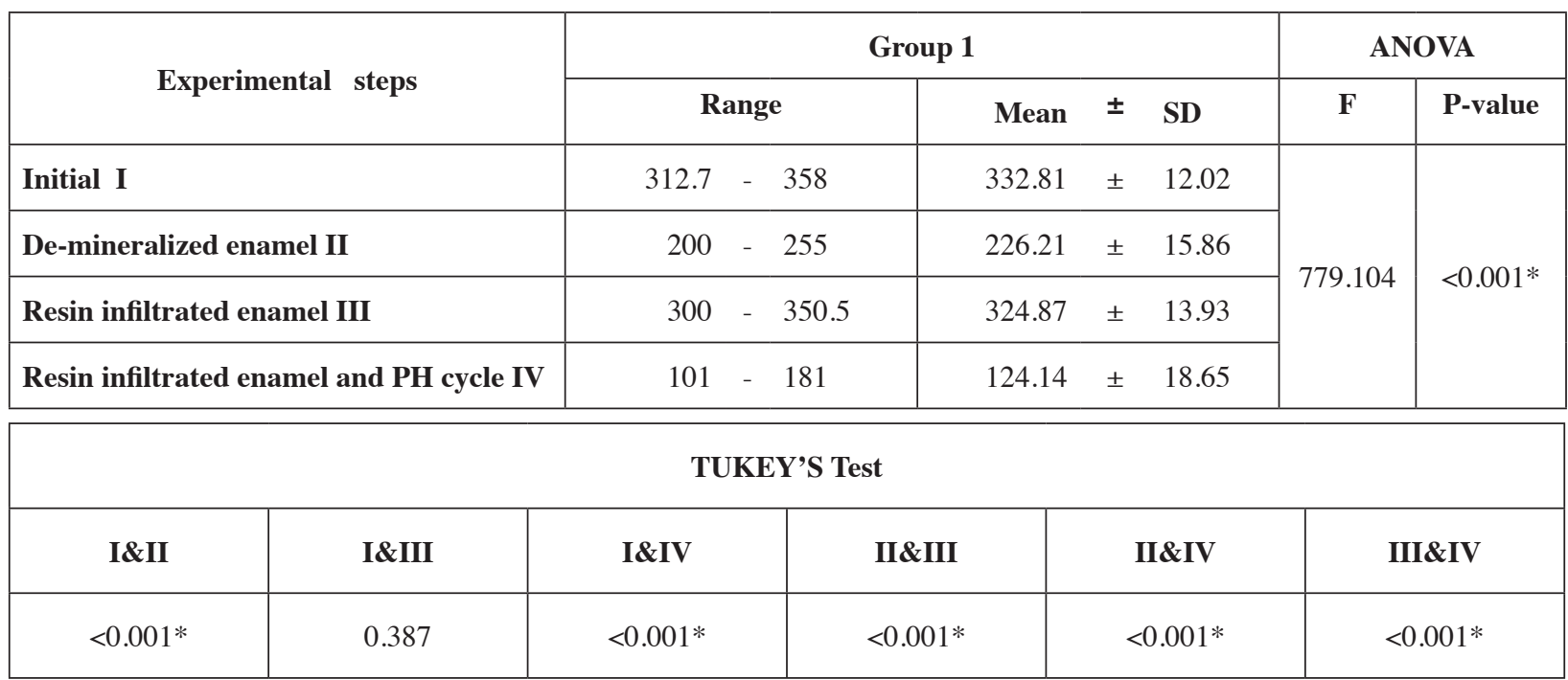


TABLE (3) Comparison of micro-hardness among different experimental steps of group 2.

\begin{tabular}{|c|c|c|c|c|c|c|c|}
\hline \multirow{2}{*}{ Experimental steps } & \multicolumn{5}{|c|}{ Group 2} & \multirow{2}{*}{$\frac{\text { ANOVA }}{\text { F }}$} & \multirow[b]{2}{*}{ P-value } \\
\hline & \multicolumn{2}{|c|}{ Range } & Mean & \pm & SD & & \\
\hline Initial I & 309 & 353 & 334.78 & \pm & 13.98 & \multirow{4}{*}{749.307} & \multirow{4}{*}{$<0.001^{*}$} \\
\hline De-mineralized enamel II & 200 & 253 & 224.96 & \pm & 18.48 & & \\
\hline Fluoride varnish III & 209 & 300.5 & 239.15 & \pm & 20.74 & & \\
\hline Fluoride varnish and $\mathrm{PH}$ cycle IV & 89 & 100.3 & 95.23 & \pm & 3.93 & & \\
\hline
\end{tabular}

\begin{tabular}{|c|c|c|c|c|c|}
\hline \multicolumn{7}{|c|}{ TUKEY'S Test } \\
\hline I\&II & I\&III & I\&IV & II\&III & II\&IV & III\&IV \\
\hline$<0.001^{*}$ & $<0.001^{*}$ & $<0.001^{*}$ & $0.033^{*}$ & $<0.001 *$ & $<0.001 *$ \\
\hline
\end{tabular}

\section{DISCUSSION}

Fluoride varnish has been used as a replacement to traditional topical fluorides ${ }^{[33]}$. It is an efficiently applied adherent material which consists of a high concentration of fluoride as a salt or silane preparation in a fast drying, alcohol, and resinbased solution ${ }^{[34]}$.

Fluoride varnish is commonly used as a caries defensive method for many years. It is considered as a physical barrier, so it prevents the direct contact between acids and enamel. In addition, it discharges fluoride in the oral cavity ${ }^{[35]}$. The varnish is distinguished from other fluoride products as easy to use, safe and effective method. Fluoride varnish can be applied every three or six month to increase its efficiency. This is one of its main drawbacks ${ }^{[36]}$.

Fluoride applies its anti-caries effect with three different mechanisms. First, it enters the fluoride ions in dental tissues and forms fluoroapatite by the present calcium and phosphate ions in saliva ${ }^{[37]}$. This insoluble sediment replaces the dissolving salts containing manganese and carbonate, which were lost during de-mineralization by bacteria. This replacement process increases the enamel resistance to acid. Second, non-cavitated lesions are re-mineralized through a similar process. Third, fluoride has antimicrobial activity ${ }^{[38]}$.
Resin infiltration is a treatment option developed to prevent the progression of initial carious lesions. The resin infiltration concept depends upon arresting the incipient enamel caries lesions by penetrating the low-viscosity resin into the enamel and occluding the diffusion pathways for acids and dissolved minerals in the enamel ${ }^{[39]}$. In addition, the resin matrix can strengthen the enamel structure mechanically, and therefore preventing breakdown of the enamel surface ${ }^{[9]}$.

Hardness, which could be defined as the resistance of a material or surface against indentation or penetration, is an important mechanical property from a biomedical engineering standpoint ${ }^{[40]}$. Measurement of micro-hardness is a suitable method to determine the strength of a hard tissue as enamel ${ }^{[41]}$. This method of assessment is fairly simple with accurate measurement and is noninvasive but needs special preparation of the samples for accurate measurements, prismless enamel layer should be removed and we get this through preparing a flat polished enamel surface ${ }^{[42]}$.

High levels of mineral content in enamel are related to abrasion resistance giving the importance of surface enamel in progression of dental decay. The objectives of this study was to evaluate microhardness of an intact enamel surface at the baseline, 
then after creating the early enamel carious lesions and finally after treatment of lesion and subjecting to pHcycling. Thus, measuring VHN(Vickers Hardness Number) was done in four steps ${ }^{[43]}$. In both tested groups there was a significant reduction in microhardness of de-mineralized enamel when compared with the sound one due to the loss of mineral content. After treatment, the micro-hardness values were increased in both tested groups due to occlusion of micro-pores created by demineralization. In the current study, the surface micro-hardness of resininfiltrated enamel was higher than that treated with fluoride varnish. This result coincided with Aziznezhad et al ${ }^{[44]}$. Such high surface hardness is obviously not a function of the resin matrix as polymerized TEGDMA is the softest (26 VHN) among the resin polymers used in dental restorative materials ${ }^{[45]}$. The infiltration resin was designed to penetrate the porous lesions left after acid etching and to fill the voids and spaces of the de-mineralized zone in a white spot lesion, thus preventing further demineralization and lesion progression [46]. It appears that the infiltration resin was able to encapsulate the hydroxyl-apatite crystals in the white spot lesion and form a relatively uniform resin-hydroxyapatite complex that exhibits high surface hardness ${ }^{[45,47]}$.

This result indicated the ability of low-viscosity resin to fill the spaces between the remaining crystals of porous lesions and create a diffusion barrier not only at the surface, but also within the enamel lesion body ${ }^{[48]}$. Therefore, a resin-infiltrated layer should be able to strengthen the de-mineralized enamel structure and prevent further wear and cavitation ${ }^{[49]}$. Some clinical studies have reported that microinvasive caries treatment with resin infiltration was an effective and safe approach to arrest initial caries lesions and preserve de-mineralized enamel ${ }^{[9]}$.

Findings of this study agreed with those reported by Paris et al ${ }^{[29]}$ and Torres et ${ }^{2}{ }^{[30]}$ in that the micro-hardness of carious lesions was significantly improved with resin infiltration. Taher et al ${ }^{[42]}$ also indicated that enamel surfaces treated with an infiltrant showed significantly higher surface hardness than treatment with fluoride varnish.

It is argued that the micro-hardness of porous lesion bodies is increased when they are filled with a resin than when they are simply untreated or re-mineralized carious lesions. In addition the difference between micro-hardness of sound enamel showed no significant difference when compared with that of treated enamel in group 1.

Although the micro-hardness of enamel treated with fluoride varnish increased, the difference between it and sound enamel was significant. This result conveys that, the re-mineralization was incomplete and less than that achieved by resin infiltration. This may be due to the low contact time of fluoride varnish with the enamel in current study because the fluoride varnish can have chemical reaction with enamel over 24 hours. On the other hand, more frequent application of fluoride varnish can enhance the effectiveness of anti-caries properties. But, in this study, one-time use of fluoride varnish was applied for the purpose of simulating professional clinical condition.

Despite resin infiltration markedly increased the micro-hardness of de-mineralized enamel, it was unable to protect enamel from $\mathrm{pH}$ cycling. This result is compatible with previous studies ${ }^{[2,9,30]}$. On the contrary, it does not agree with the majority of studies. This may be due to the difference in $\mathrm{pH}$ cycle model where it used long-term (50 days) $\mathrm{pH}$ cycling models under weaker challenge conditions ${ }^{[9}$, ${ }^{13,24]}$ which is different from the short-term protocol used in the current study. Another reason can be the polishing procedure after application of resin infiltrant, since it was established that resin-based materials are able to defend enamel against erosion when they are found over enamel as a physical barrier ${ }^{[50]}$.

The same was found in group 2 where fluoride varnish does not provide a physical or mechanical barrier against $\mathrm{pH}$ cycle. The result is compatible with Bayrak et al., ${ }^{[51]}$ despite the difference in acidic 
challenge. Tricalcium phosphate which added to flouride varnish is a hybrid material formed by a milling technique that fuses beta tricalcium phosphate and sodium lauryl sulfate or fumaric acid ${ }^{[4]}$. This blending results in a functionalized calcium and a free phosphate, which is considered to increase fluoride retention in enamel and facilitate remineralization ${ }^{[6]}$. When tricalcium phosphate comes into contact with the tooth surface and is moistened by saliva, the protective barrier breaks down, making calcium, phosphate, and fluoride ions available to the teeth ${ }^{[5]}$.The tricalcium phosphate with fluoride can promote the protective effect of enamel against any $\mathrm{pH}$ changes. The fluoride varnish used in the current study does not contain tricalcium phosphate this may explained the results of current study. Magalhaes et al., 2007 stated that the commercial fluoride varnishes chemically reacted with enamel decreasing the softening of enamel, but this was not sufficient to decrease the enamel loss aggravated by the acidic challenge. Despite the fact that the $\mathrm{NaF}$ varnishes caused a significant reduction of softening of enamel but it might not be effective in the clinical situation. This result come in coincide with our study ${ }^{[52]}$. On the other hand many other studies showing a resistance of commercial varnishes to acidic challenge in vitro and in situ protocols ${ }^{[1,3,53]}$ this may due to uses of different challenges and different types of fluoride varnish.

The reduction in micro-hardness after $\mathrm{PH}$ cycle of resin infiltrated enamel was significantly less than recorded in group 2. the current results compatible with the result of study carried out by Aziznezhad et al. ${ }^{[44]}$. This may be attributed to the difference in chemical composition of the two tested materials.

Conclusion: Within the limitations of this study, it is concluded that the resin infiltrated enamel microhardness was higher than that of de-mineralized enamel treated with fluoride varnish before and after $\mathrm{pH}$ cycling.

\section{REFERENCES}

1. Vieira, A., et al., Inhibition of erosive wear by fluoride varnish. Caries Research, 2007. 41(1): p. 61-67.

2. Neres, É., et al., Microhardness and Roughness of Infiltrated White Spot Lesions Submitted to Different Challenges. Operative Dentistry, 2017.

3. Comar, L.P., et al., TiF4 and $\mathrm{NaF}$ varnishes as anti-erosive agents on enamel and dentin erosion progression in vitro. Journal of Applied Oral Science, 2015. 23(1): p. 14-18.

4. Rirattanapong, P., et al., Effect of various forms of calcium in dental products on human enamel microhardness in vitro. Southeast Asian Journal of Tropical Medicine and Public Health, 2012. 43(4): p. 1053.

5. Karlinsey, R.L., et al., In vitro evaluation of eroded enamel treated with fluoride and a prospective tricalcium phosphate agent. Journal of Dentistry and Oral Hygiene, 2009. 1(4): p. 052-058.

6. Shellis, R., et al., Methodology and models in erosion research: discussion and conclusions. Caries research, 2011. 45(Suppl. 1): p. 69-77.

7. Esfahani, K.S., R. Mazaheri, and L. Pishevar, Effects of treatment with various remineralizing agents on the microhardness of demineralized enamel surface. Journal of dental research, dental clinics, dental prospects, 2015.9(4): p. 239.

8. Mohd Said, S.N., M. Ekambaram, and C.K. Yiu, Effect of different fluoride varnishes on remineralization of artificial enamel carious lesions. International Journal of Paediatric Dentistry, 2017. 27(3): p. 163-173.

9. Paris, S., et al., Micro-hardness and mineral loss of enamel lesions after infiltration with various resins: influence of infiltrant composition and application frequency in vitro. Journal of dentistry, 2013. 41(6): p. 543-548.

10. Horuztepe, S.A. and M. Baseren, Effect of resin infiltration on the color and microhardness of bleached white-spot lesions in bovine enamel (an in vitro study). Journal of Esthetic and Restorative Dentistry, 2017.

11. Ionta, F.Q., et al., Penetration of resin-based materials into initial erosion lesion: A confocal microscopic study. Microscopy research and technique, 2016. 79(2): p. 72-80.

12. Gajewski, V.E., et al., Monomers used in resin composites: degree of conversion, mechanical properties and water sorption/solubility. Brazilian dental journal, 2012. 23(5): p. 508-514. 
13. Gelani, R., et al., In vitro progression of artificial white spot lesions sealed with an infiltrant resin. Operative dentistry, 2014. 39(5): p. 481-488.

14. Paris, S., et al., Comparison of sealant and infiltrant penetration into pit and fissure caries lesions in vitro. Journal of dentistry, 2014. 42(4): p. 432-438.

15. Belli, R., et al., Wear and morphology of infiltrated white spot lesions. Journal of dentistry, 2011. 39(5): p. 376-385.

16. Zhao, X. and Y. Ren, Surface properties and color stability of resin-infiltrated enamel lesions. Operative dentistry, 2016. 41(6): p. 617-626.

17. Altarabulsi, M., et al., Clinical safety, quality and effect of resin infiltration for proximal caries. Eur J Paediatr Dent, 2014. 15(1): p. 39-44.

18. Senestraro, S.V., et al., Minimally invasive resin infiltration of arrested white-spot lesions: a randomized clinical trial. The Journal of the American Dental Association, 2013. 144(9): p. 997-1005.

19. Borges, A., et al., Is resin infiltration an effective esthetic treatment for enamel development defects and white spot lesions? A systematic review. Journal of dentistry, 2017. 56: p. 11-18.

20. Arslan, S., et al., Effect of resin infiltration on enamel surface properties and Streptococcus mutans adhesion to artificial enamel lesions. Dental materials journal, 2015. 34(1): p. 25-30.

21. Urabe, I., et al., Physical properties of the dentin-enamel junction region. American Journal of Dentistry, 2000. 13(3): p. 129-135.

22. LEVY, F., et al., The erosion and abrasion-inhibiting effect of TiF4 and NaF varnishes and solutions on enamel in vitro. International journal of paediatric dentistry, 2012. 22(1): p. 11-16.

23. Catelan, A., et al., Effect of artificial aging on the roughness and microhardness of sealed composites. Journal of Esthetic and Restorative Dentistry, 2010. 22(5): p. 324-330.

24. Buzalaf, M.A.R., et al., pH-cycling models for in vitro evaluation of the efficacy of fluoridated dentifrices for caries control: strengths and limitations. Journal of Applied Oral Science, 2010. 18(4): p. 316-334.

25. Queiroz, C.S., et al., pH-cycling models to evaluate the effect of low fluoride dentifrice on enamel de-and remineralization. Brazilian Dental Journal, 2008. 19(1): p. 21-27.
26. Zhao, X., et al., Effectiveness of resin-based materials against erosive and abrasive enamel wear. Clinical oral investigations, 2017. 21(1): p. 463-468.

27. EL-WASSEFY, N.A., Remineralizing effect of cold plasma and/or bioglass on demineralized enamel. Dental materials journal, 2017.36(2): p. 157-167.

28. Gurdogan, E.B., D. Ozdemir-Ozenen, and N. Sandalli, Evaluation of Surface Roughness Characteristics Using Atomic Force Microscopy and Inspection of Microhardness Following Resin Infiltration with Icon ${ }^{\circledR}$. Journal of Esthetic and Restorative Dentistry, 2017. 29(3): p. 201-208.

29. Paris, S., W. Hopfenmuller, and H. Meyer-Lueckel, Resin infiltration of caries lesions: an efficacy randomized trial. Journal of dental research, 2010. 89(8): p. 823-826.

30. Torres, C., et al., Effect of caries infiltration technique and fluoride therapy on microhardness of enamel carious lesions. Operative dentistry, 2012. 37(4): p. 363-369.

31. Oliveira, G., et al., In vitro effects of resin infiltration on enamel erosion inhibition. Operative dentistry, 2015. 40(5): p. 492-502.

32. Vicente, A., et al., Efficacy of fluoride varnishes for preventing enamel demineralization after interproximal enamel reduction. Qualitative and quantitative evaluation. PloS one, 2017. 12(4): p. e0176389.

33. Delbem, A.C.B., et al., Effect of fluoridated varnish and silver diamine fluoride solution on enamel demineralization: pH-cycling study. Journal of Applied Oral Science, 2006. 14(2): p. 88-92.

34. Shafi, I., Fluoride varnish reduces white spot lesions during orthodontic treatment. Evidence-based dentistry, 2008. 9(3): p. 81-81.

35. Muñoz-Millán, P., et al., Effectiveness of fluoride varnish in preventing early childhood caries in rural areas without access to fluoridated drinking water: A randomized control trial. Community Dentistry and Oral Epidemiology, 2017.

36. Chu, C. and E. Lo, Uses of sodium fluoride varnish in dental practice. Annals of the Royal Australasian College of Dental Surgeons, 2008. 19: p. 58.

37. Buzalaf, M.A.R., et al., Mechanisms of action of fluoride for caries control, in Fluoride and the oral environment. 2011, Karger Publishers. p. 97-114.

38. Ten Cate, J., Contemporary perspective on the use of fluoride products in caries prevention. British dental journal, 2013. 214(4): p. 161-167. 
39. Crombie, F., et al., Resin infiltration of developmentally hypomineralised enamel. International journal of paediatric dentistry, 2014. 24(1): p. 51-55.

40. Mahoney, E., et al., The hardness and modulus of elasticity of primary molar teeth: an ultra-micro-indentation study. Journal of dentistry, 2000. 28(8): p. 589-594.

41. Mahoney, E.K., et al., Mechanical properties and microstructure of hypomineralised enamel of permanent teeth. Biomaterials, 2004. 25(20): p. 5091-5100.

42. Taher, N.M., H.A. Alkhamis, and S.M.l. Dowaidi, The influence of resin infiltration system on enamel microhardness and surface roughness: An in vitro study. The Saudi Dental Journal, 2012. 24(2): p. 79-84.

43. Mueller, J., et al., Surface tridimensional topography analysis of materials and finishing procedures after resinous infiltration of subsurface bovine enamel lesions. Quintessence international, 2011.42(2).

44. Aziznezhad, M., et al., Comparison of the effect of resin infiltrant, fluoride varnish, and nano-hydroxy apatite paste on surface hardness and streptococcus mutans adhesion to artificial enamel lesions. Electronic physician, 2017. 9(3): p. 3934

45. Venter, S.A.d.S., et al., Hardness and degree of conversion of dental restorative composites based on an organicinorganic hybrid. Materials Research, 2013. 16(4): p. 898902.
46. Paris, S. and H. Meyer-Lueckel, Masking of labial enamel white spot lesions by resin infiltration--A clinical report. Quintessence international, 2009.40(9).

47. Paris, S., et al., Penetration coefficients of commercially available and experimental composites intended to infiltrate enamel carious lesions. Dental Materials, 2007. 23(6): p. 742-748.

48. Baek, C.-J., et al., The effects of light intensity and light-curing time on the degree of polymerization of dental composite resins. Dental materials journal, 2008. 27(4): p. 523-533.

49. Tuncer, S., et al., The effect of a modeling resin and thermocycling on the surface hardness, roughness, and color of different resin composites. Journal of Esthetic and Restorative Dentistry, 2013. 25(6): p. 404-419.

50. Tereza, G.P.G., et al., Influence of removing excess of resin-based materials applied to eroded enamel on the resistance to erosive challenge. Journal of dentistry, 2016. 47: p. 49-54.

51. Bayrak, S., et al., Effect of Fluoride Varnish Containing CPP-ACP on Preventing Enamel Erosion. Scanning, 2017. 2017.

52. Magalhaes, A., et al., Effect of an experimental $4 \%$ titanium tetrafluoride varnish on dental erosion by a soft drink. Journal of Dentistry, 2007. 35(11): p. 858-861.

53. Murakami, C., et al., Effect of fluoride varnish and gel on dental erosion in primary and permanent teeth. Archives of oral biology, 2009. 54(11): p. 997-1001. 